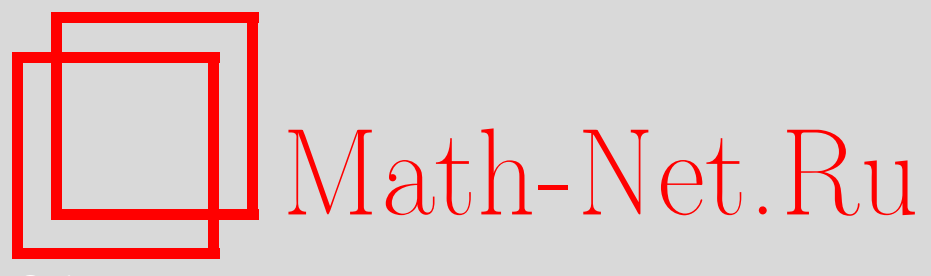

М. М. Маламуд, В. И. Могилевский, С. Хасси, Об унитарной эквивалентности собственных расширений эрмитова оператора и функции Вейля, Матем. заметки, 2012, том 91, выпуск 2, 316-320

DOI: https://doi.org/10.4213/mzm9024

Использование Общероссийского математического портала Math-Net.Ru подразумевает, что вы прочитали и согласны с пользовательским соглашением http://www . mathnet.ru/rus/agreement

Параметры загрузки:

IP: 54.224 .135 .184

26 апреля 2023 г., 18:16:43

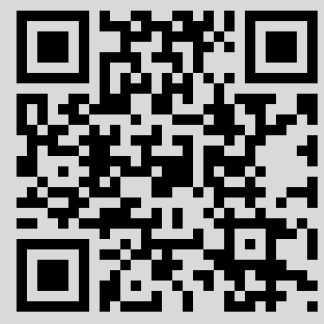




\section{Об унитарной эквивалентности собственных расширений эрмитова оператора и функции Вейля}

\section{М. М. Маламуд, В. И. Могилевский, С. Хасси}

1. Введение. Пусть $A$ - симметрический плотно заданный оператор в гильбертовом пространстве $\mathfrak{H}$ с равными индексами дефекта. В последние 30 лет заметную популярность в теории расширений приобрел подход, основанный на понятиях граничной тройки оператора $A^{*}$ и соответствующей функции Вейля (см. [1]-[3]). Хорошо известно (см. [2]), что в случае простого $A$ функция Вейля $M(\cdot)$ граничной тройки $\Pi=\left\{\mathscr{H}, \Gamma_{0}, \Gamma_{1}\right\}$ определяет ее однозначно, с точностью до унитарной эквивалентности. В частности, $M(\cdot)$ определяет пару $\left\{A, A_{0}\right\}$, в которой $A_{0}:=A^{*}\left\lceil\operatorname{ker} \Gamma_{0}=A_{0}^{*}\right.$ однозначно с точностью до унитарного подобия.

С другой стороны, если $\Pi_{D}=\left\{\mathscr{H}_{0} \oplus \mathscr{H}_{1}, \Gamma, \Gamma^{\top}\right\}-$ граничная тройка дуальной пары $\left\{A, A^{\top}\right\}$ операторов, то соответствующая функция Вейля определяет тройку $\Pi_{D}$ только с точностью до слабого подобия (см. [4]). В недавних работах [5]-[7] найдены условия на пару $\left\{A, A^{\top}\right\}$, при выполнении которых функция Вейля определяет тройку $\Pi_{D}$ с точностью до подобия.

В настоящей заметке исследуются дуальные пары $\left\{A, A^{\top}\right\}$, в которых $A=A^{\top}$, и те их граничные тройки, функции Вейля которых имеют вид $\widetilde{M}(\cdot)=K^{*}(B-M(\cdot))^{-1} K($ см. ниже формулу (4)).

Найдены достаточные условия на один из операторов $A_{0}$ или $A_{B}:=A^{*}\left\lceil\operatorname{ker}\left(\Gamma_{1}-B \Gamma_{0}\right)\right.$, при которых функция Вейля $\widetilde{M}(\cdot)$ определяет пару $\left\{A_{0}, A_{B}\right\}$ (да и всю тройку $\Pi_{D}$ ) однозначно (с точностью до унитарного подобия). Показано также, что в других случаях функция Вейля $\widetilde{M}(\cdot)$ не определяет основной оператор $A_{B}$ даже с точностью до подобия.

Всюду далее $\mathfrak{H}, \mathscr{H}_{j}$ - гильбертовы пространства, $\left[\mathscr{H}_{1}, \mathscr{H}_{2}\right]-$ множество ограниченных линейных операторов из $\mathscr{H}_{1}$ в $\mathscr{H}_{2},[\mathscr{H}]:=[\mathscr{H}, \mathscr{H}], \mathscr{C}(\mathfrak{H})-$ множество замкнутых плотно заданных операторов в $\mathfrak{H}$.

2. Граничные тройки и функция Вейля. Говорят, что операторы $A, A^{\top} \in \mathscr{C}(\mathfrak{H})$ образуют дуальную пару (ДП) $\left\{A, A^{\top}\right\}$, если $(A f, g)=\left(f, A^{\top} g\right)$ для всех $f \in \operatorname{dom}(A), g \in$ $\operatorname{dom}\left(A^{\top}\right)$.

ОПредЕлЕНиЕ 1 [8]. Пусть $\mathscr{H}_{0}, \mathscr{H}_{1}$ - гильбертовы пространства и

$$
\Gamma=\left(\begin{array}{c}
\Gamma_{0} \\
\Gamma_{1}
\end{array}\right): \operatorname{dom}\left(\left(A^{\top}\right)^{*}\right) \rightarrow \mathscr{H}_{0} \oplus \mathscr{H}_{1}, \quad \Gamma^{\top}=\left(\begin{array}{l}
\Gamma_{0}^{\top} \\
\Gamma_{1}^{\top}
\end{array}\right): \operatorname{dom}\left(A^{*}\right) \rightarrow \mathscr{H}_{1} \oplus \mathscr{H}_{0}
$$

- линейные отображения. Совокупность $\Pi_{D}=\left\{\mathscr{H}_{0} \oplus \mathscr{H}_{1}, \Gamma, \Gamma^{\top}\right\}$ называют граничной тройкой для дуальной пары $\left\{A, A^{\top}\right\}$, если отображения $\Gamma, \Gamma^{\top}$ сюръективны и для всех $f \in \operatorname{dom}\left(\left(A^{\top}\right)^{*}\right), g \in \operatorname{dom}\left(A^{*}\right)$ выполняется тождество Грина

$$
\left(\left(A^{\top}\right)^{*} f, g\right)-\left(f, A^{*} g\right)=\left(\Gamma_{1} f, \Gamma_{0}^{\top} g\right)-\left(\Gamma_{0} f, \Gamma_{1}^{\top} g\right) .
$$

Граничная тройка для ДП $\left\{A, A^{\top}\right\}$ существует всегда и не единственна ([4], [8]). С каждой тройкой $\Pi_{D}$ связаны два расширения оператора $A$

$$
A_{j}=\left(A^{\top}\right)^{*}\left\lceil\operatorname{ker} \Gamma_{j}, \quad j \in\{0,1\} .\right.
$$

Оператор-функцию $F: \mathbb{C} \backslash \mathbb{R} \rightarrow[\mathscr{H}]$ называют неванлинновской (и относят к классу $R[\mathscr{H}])$, если $\operatorname{Im} z \operatorname{Im} F(z) \geqslant 0$ и $F^{*}(z)=F(\bar{z}), z \in \mathbb{C} \backslash \mathbb{R}$. 
ОПределение 2 [4]. Пусть $\Pi_{D}=\left\{\mathscr{H}_{0} \oplus \mathscr{H}_{1}, \Gamma, \Gamma^{\top}\right\}$ - граничная тройка для дуальной пары $\left\{A, A^{\top}\right\}$. Голоморфная оператор-функция $M(\cdot): \rho\left(A_{0}\right) \rightarrow\left[\mathscr{H}_{0}, \mathscr{H}_{1}\right]$, определенная равенством

$$
M(z) \Gamma_{0} f_{z}=\Gamma_{1} f_{z}, \quad f_{z} \in \mathfrak{N}_{z}\left(A^{\top}\right)=\operatorname{ker}\left(\left(A^{\top}\right)^{*}-z\right),
$$

называется функиией Вейля, соответствующей граничной тройке $\Pi_{D}$.

Пусть, далее, $A \in \mathscr{C}(\mathfrak{H})$ - симметрический оператор в $\mathfrak{H}$ с равными дефектными числами $n_{ \pm}(A)$. Положим $A^{\top}:=A$ и рассмотрим ДП $\{A, A\}$. Полагая для этой пары в определении $1 \mathscr{H}_{1}=\mathscr{H}_{0}=: \mathscr{H}, \Gamma_{j}^{\top}=\Gamma_{j}, j \in\{0,1\}$, получим граничную тройку $\Pi=\left\{\mathscr{H}, \Gamma_{0}, \Gamma_{1}\right\}$ для $A^{*}$ в смысле [1]. В этом случае тождество (1) принимает вид

$$
\left(A^{*} f, g\right)-\left(f, A^{*} g\right)=\left(\Gamma_{1} f, \Gamma_{0} g\right)_{\mathscr{H}}-\left(\Gamma_{0} f, \Gamma_{1} g\right), \quad f, g \in \operatorname{dom}\left(A^{*}\right),
$$

а формула (2) задает самосопряженные расширения $A_{j}=A^{*}\left\lceil\operatorname{ker} \Gamma_{j}\right.$. При этом, функция Вейля $M(\cdot)$, введенная в [2], принадлежит классу $R[\mathscr{H}]$.

ПреДЛОЖенИе 1 [4]. Пусть $\Pi=\left\{\mathscr{H}, \Gamma_{0}, \Gamma_{1}\right\}$ - граничная тройка для $A^{*}$ и $M(\cdot)$ соответствующая функиия Вейля. Пусть также $B, K \in[\mathscr{H}], 0 \in \rho(K)$ u

$$
\widetilde{\Gamma}=\left(\begin{array}{l}
\widetilde{\Gamma}_{0} \\
\widetilde{\Gamma}_{1}
\end{array}\right), \quad \widetilde{\Gamma}^{\top}=\left(\begin{array}{c}
\widetilde{\Gamma}_{0}^{\top} \\
\widetilde{\Gamma}_{1}^{\top}
\end{array}\right),
$$

где

$$
\widetilde{\Gamma}_{0}=K^{-1}\left(B \Gamma_{0}-\Gamma_{1}\right), \quad \widetilde{\Gamma}_{1}=K^{*} \Gamma_{0} ; \quad \widetilde{\Gamma}_{0}^{\top}=K^{-1}\left(B^{*} \Gamma_{0}-\Gamma_{1}\right), \quad \widetilde{\Gamma}_{1}^{\top}=K^{*} \Gamma_{0} .
$$

Тогда $\widetilde{\Pi}_{D}=\left\{\mathscr{H} \oplus \mathscr{H}, \widetilde{\Gamma}, \widetilde{\Gamma}^{\top}\right\}-$ граничная тройка для ДП $\{A, A\}$ такая, что

$$
\widetilde{A}_{0}\left(=A^{*}\left\lceil\operatorname{ker} \widetilde{\Gamma}_{0}\right)=A^{*}\left\lceil\operatorname{ker}\left(\Gamma_{1}-B \Gamma_{0}\right),\right.\right.
$$

и функиия Вейля для $\widetilde{\Pi}_{D}$ имеет вид

$$
\widetilde{M}(z)=K^{*}(B-M(z))^{-1} K, \quad z \in \rho\left(\widetilde{A}_{0}\right) .
$$

3. Основная лемма. Каждая функция $F(\cdot) \in R[\mathscr{H}]$ допускает интегральное представление

$$
F(z)=C+D z+\int_{\mathbb{R}}\left(\frac{1}{t-z}-\frac{t}{1+t^{2}}\right) d \Sigma(t),
$$

в котором $C=C^{*}, D \in[\mathscr{H}], D \geqslant 0$ и $\Sigma(\cdot): \mathbb{R} \rightarrow[\mathscr{H}]$ - неубывающая операторно-значная функция,

$$
\Sigma(t-0)=\Sigma(t) \quad \text { и } \quad \int_{\mathbb{R}} \frac{d \Sigma(t)}{1+t^{2}} \in[\mathscr{H}]
$$

(см. [9]). Функцию $\Sigma(\cdot)$ называют спектральной мерой функции $F(\cdot)$, а $C$ и $D$ - nараметрами.

Согласно теореме Лебега мера $\Sigma(\cdot)$ допускает разложение

$$
\Sigma(t)=\Sigma^{\mathrm{ac}}(t)+\Sigma^{\mathrm{s}}(t),
$$

в котором $\Sigma^{\text {ac }}(\cdot)$ и $\Sigma^{\mathrm{S}}(\cdot)$ - абсолютно непрерывная и сингулярная компоненты меры $\Sigma(\cdot)$ соответственно.

Лемма 1. Пусть $F_{j}(\cdot) \in R[\mathscr{H}]$ u $\Sigma_{j}(\cdot), C_{j}, D_{j}$ - спектральная мера и параметры функиии $F_{j}(\cdot), j \in\{1,2\}$, соответственно. Пусть также $B_{1}, B_{2} \in[\mathscr{H}]$ u $B_{1} \neq B_{1}^{*}$, $B_{2} \neq B_{2}^{*} u$

$$
\Omega_{+}:=\left\{z \in \mathbb{C}: 0 \in \rho\left(B_{1}-F_{1}(z)\right) \cap \rho\left(B_{2}-F_{2}(z)\right)\right\} \cap \mathbb{C}_{+} \neq \varnothing .
$$


Если существуют $K_{1}, K_{2} \in[\mathscr{H}]$ такие, что $0 \in \rho\left(K_{1}\right) \cap \rho\left(K_{2}\right)$ u

$$
K_{1}^{*}\left(B_{1}-F_{1}(z)\right)^{-1} K_{1}=K_{2}^{*}\left(B_{2}-F_{2}(z)\right)^{-1} K_{2}, \quad z \in \Omega_{+},
$$

то справедливы равенства

$$
\begin{gathered}
\widetilde{\Sigma}_{1}^{\mathrm{s}}(t)=\widetilde{\Sigma}_{2}^{\mathrm{s}}(t), \quad \widetilde{\Sigma}_{2}^{\mathrm{ac}}(t)-\widetilde{\Sigma}_{1}^{\mathrm{ac}}(t)=\frac{1}{\pi} t\left(\operatorname{Im} \widetilde{B}_{2}-\operatorname{Im} \widetilde{B}_{1}\right), \\
\widetilde{C}_{1}-\operatorname{Re} \widetilde{B}_{1}=\widetilde{C}_{2}-\operatorname{Re} \widetilde{B}_{2}, \quad \widetilde{D}_{1}=\widetilde{D}_{2},
\end{gathered}
$$

в которых $K_{j}^{-*}:=\left(K_{j}^{-1}\right)^{*}, \widetilde{D}_{j}:=K_{j}^{-1} D_{j} K_{j}^{-*} u$

$$
\widetilde{\Sigma}_{j}(\cdot):=K_{j}^{-1} \Sigma_{j}(\cdot) K_{j}^{-*}, \quad \widetilde{B}_{j}:=K_{j}^{-1} B_{j} K_{j}^{-*}, \quad \widetilde{C}_{j}:=K_{j}^{-1} C_{j} K_{j}^{-*}, \quad j \in\{1,2\} .
$$

\section{4. Достаточные условия унитарной эквивалентности.}

ОПредЕлениЕ 3. Пусть $A^{(j)} \in \mathscr{C}\left(\mathfrak{H}_{j}\right), j \in\{1,2\},-$ симметрические операторы и $\Pi_{j}=$ $\left\{\mathscr{H}_{j}, \Gamma_{0}^{(j)}, \Gamma_{1}^{(j)}\right\}$ - граничная тройка для $\left(A^{(j)}\right)^{*}$. Говорят, что тройки $\Pi_{1}$ и $\Pi_{2}$ унитарно эквивалентны, если существует унитарный оператор $U \in\left[\mathfrak{H}_{1}, \mathfrak{H}_{2}\right]$ такой, что

$$
U A^{(1)}=A^{(2)} U \quad \text { и } \quad \Gamma_{j}^{(2)} U=\Gamma_{j}^{(1)}, \quad j \in\{0,1\} .
$$

Если тройки $\Pi_{1}$ и $\Pi_{2}$ унитарно эквивалентны и $\mathscr{H}=\mathscr{H}_{1}=\mathscr{H}_{2}$, то для каждого $B \in$ $\mathscr{C}(\mathscr{H})$ справедливы равенства $U A_{B}^{(1)}=A_{B}^{(2)} U$, в которых

$$
A_{B}^{(j)}=\left(A^{(j)}\right)^{*}\left\lceil\operatorname{dom}\left(A_{B}^{(j)}\right), \quad \operatorname{dom}\left(A_{B}^{(j)}\right)=\operatorname{ker}\left(\Gamma_{1}^{(j)}-B \Gamma_{0}^{(j)}\right), \quad j \in\{1,2\} .\right.
$$

В частности, пары $\left\{A_{0}^{(1)}, A_{1}^{(1)}\right\}$ и $\left\{A_{0}^{(2)}, A_{1}^{(2)}\right\}$ унитарно эквивалентны.

Напомним, что симметрический оператор $A$ называют простым, если он не допускает представления $A=S_{1} \oplus S_{2}$, в котором $S_{1}=S_{1}^{*} \neq 0$ и $S_{2} \subset S_{2}^{*}$.

В случае простого $A$ функция Вейля $M(\cdot)$ определяет граничную тройку для $A^{*}$ однозначно с точностью до унитарной эквивалентности [2], [3].

ПредЛОЖенИЕ 2. Пусть $A^{(j)} \in \mathscr{C}\left(\mathfrak{H}_{j}\right)$ - простой симметрический оператор, $\Pi_{j}=$ $\left\{\mathscr{H}_{j}, \Gamma_{0}^{j}, \Gamma_{1}^{j}\right\}$ - граничная тройка для $\left(A^{(j)}\right)^{*}$ и $M_{j}(\cdot)$ - соответствующая функиия Вейля, $j \in\{1,2\}$. Пусть $\Sigma_{j}(\cdot)$ - спектральная мера $u C_{j}=C_{j}^{*}, D_{j}=D_{j}^{*} \geqslant 0$ - параметры $R_{\mathscr{H}}$-функиии $M_{j}(\cdot), j \in\{1,2\}$ (см. (5)). Пусть, далее, $B_{j} \neq B_{j}^{*} \in\left[\mathscr{H}_{j}\right], A_{B_{j}}^{(j)}$ определены равенствами (10) и

$$
\Omega_{+}:=\rho\left(A_{B_{1}}\right) \cap \rho\left(A_{B_{2}}\right) \cap \mathbb{C}_{+} \neq \varnothing .
$$

Если $K_{j} \in\left[\mathscr{H}, \mathscr{H}_{j}\right], 0 \in \rho\left(K_{j}\right), j \in\{1,2\}, u$

$$
K_{1}^{*}\left(B_{1}-M_{1}(z)\right)^{-1} K_{1}=K_{2}^{*}\left(B_{2}-M_{2}(z)\right)^{-1} K_{2}, \quad z \in \Omega_{+},
$$

то справедливы равенства (9).

Следствие 1. Пусть в условиях предложения $2 \operatorname{Im} B_{j}=K_{j} J K_{j}^{*}$, где $J=J^{*}=J^{-1} u$ $0 \in \rho\left(K_{j}\right), j \in\{1,2\}$. Тогда операторы $A_{B_{1}}^{(1)} \in \operatorname{Ext}_{A^{(1)}} u A_{B_{2}}^{(2)} \in \operatorname{Ext}_{A^{(2)}}$ унитарно подобнъ.

НАБросоК дОКАЗАТЕльствА. В силу условий теоремы, $\operatorname{Im} \widetilde{B}_{j}=K_{j}^{-1} \operatorname{Im} B_{j} K_{j}^{-*}=J$, $j \in\{1,2\}$. Поэтому равенства (9) дают $\widetilde{\Sigma}_{1}^{\text {ac }}(t)=\widetilde{\Sigma}_{2}^{\text {ac }}(t)$ и $\widetilde{\Sigma}_{1}^{\mathrm{s}}(t)=\widetilde{\Sigma}_{2}^{\mathrm{s}}(t), t \in \mathbb{R}$. Отсюда уже легко вытекает унитарная эквивалентность.

ЗАмечание 1. Согласно [3; теорема 7.1] одна из характеристических оператор-функций (почти разрешимого) расширения $A_{B_{j}}^{(j)} \in \operatorname{Ext}_{A^{(j)}}$ имеет вид

$$
W_{j}(z)=I_{\mathscr{C}}+2 i K_{j}^{*}\left(B_{j}^{*}-M_{j}(z)\right)^{-1} K_{j} J, \quad j \in\{1,2\} .
$$

Поэтому равенство (11) эквивалентно равенству $W_{1}(z)=W_{2}(z), \bar{z} \in \Omega_{+}$, и следствие 1 дает другое доказательство теоремы об унитарном подобии расширений в случае совпадения их характеристических функций (см. [9], [3]). 
Для формулировки следующего результата наряду с тройками $\Pi_{1}$ и $\Pi_{2}$ мы введем граничные тройки $\widehat{\Pi}_{j}=\left\{\mathscr{H}, \widehat{\Gamma}_{0}^{j}, \widehat{\Gamma}_{1}^{j}\right\}, j \in\{0,1\}$, для $A^{*}$, полагая

$$
\widehat{\Gamma}_{0}^{j}:=K_{j}^{-1}\left(\left(\operatorname{Re} B_{j}\right) \Gamma_{0}^{j}-\Gamma_{1}^{j}\right), \quad \widehat{\Gamma}_{1}^{j}:=K_{j}^{*} \Gamma_{0}^{j}, \quad j \in\{1,2\} .
$$

Теорема 1. Пусть в условиях предложения 2 каждая из операторных мер $\Sigma_{j}^{\mathrm{ac}}, j \in$ $\{1,2\}$, не эквивалентна операторной мере Лебега $I_{\mathscr{H}} d t$. Тогда граничные тройки $\widehat{\Pi}_{1} u$ $\widehat{\Pi}_{2}$ унитарно эквивалентны. В частности, расширения $A_{B_{1}}^{(1)} \in \operatorname{Ext}_{A^{(1)}} u A_{B_{2}}^{(2)} \in \operatorname{Ext}_{A^{(2)}}$ унитарно эквивалентны.

Следствие 2. Пусть в условиях предложения $2 \sigma_{\text {ас }}\left(A_{0}^{(1)}\right) \cup \sigma_{\text {ac }}\left(A_{0}^{(2)}\right) \neq \mathbb{R}$. Тогда граничные тройки $\widehat{\Pi}_{1} u \widehat{\Pi}_{2}$ унитарно эквивалентны. В частности, операторы $A_{B_{1}}^{(1)} \in \operatorname{Ext}_{A^{(1)}}$ и $A_{B_{2}}^{(2)} \in \operatorname{Ext}_{A^{(2)}}$ унитарно подобнъ.

Условия следствия 2 выполнены, например, если $\rho\left(A_{B_{1}}^{(1)}\right) \cap \rho\left(A_{B_{2}}^{(2)}\right) \cap \mathbb{R} \neq \varnothing$.

Теорема 2. Пусть выполнены условия предложения 2 и

$$
\Omega_{ \pm}:=\rho\left(A_{B_{1}}^{(1)}\right) \cap \rho\left(A_{B_{2}}^{(2)}\right) \cap \mathbb{C}_{ \pm} \neq \varnothing .
$$

Если равенство (11) выполняется в окрестностях некоторых точек $z_{ \pm} \in \Omega_{ \pm}$, то граничные тройки $\widehat{\Pi}_{1} u \widehat{\Pi}_{2}$ унитарно эквивалентны. В частности, расширения $A_{B_{1}}^{(1)} \in \operatorname{Ext}_{A^{(1)}} u$ $A_{B_{2}}^{(2)} \in \operatorname{Ext}_{A^{(2)}}$ унитарно подобны.

\section{5. Негативные результаты.}

Tеорема 3. Пусть $A^{(1)} \in \mathscr{C}(\mathfrak{H})$ - простой симметрический оператор, $\Pi_{1}=\left\{\mathscr{H}, \Gamma_{0}^{(1)}\right.$, $\left.\Gamma_{1}^{(1)}\right\}$ - граничная тройка для $\left(A^{(1)}\right)^{*}, M_{1}(\cdot)$ - соответствующая функция Вейля $и\left(B_{1}^{*} \neq\right)$ $B_{1} \in\left[\mathscr{H}_{1}\right]$ - аккумулятивный оператор. Тогда существует (не единственный) простой симметрический оператор $A^{(2)} \in \mathscr{C}(\mathfrak{H})$, граничная тройка $\Pi_{2}=\left\{\mathscr{H}, \Gamma_{0}^{(2)}, \Gamma_{1}^{(2)}\right\}$ для $A^{(2) *}$ $u$ (неединственный) диссипативный оператор $B_{2} \in[\mathscr{H}]$ такой, что

$$
\left(B_{1}-M_{1}(z)\right)^{-1}=\left(B_{2}-M_{2}(z)\right)^{-1}, \quad z \in \mathbb{C}_{+},
$$

но граничные тройки $\Pi_{1} u \Pi_{2}$ не унитарно эквивалентны. Более того, операторы $A_{B_{1}}^{(1)} u$ $A_{B_{2}}^{(2)}$ вида (10) не подобны.

ПримеР 1. Рассмотрим в $L^{2}(\mathbb{R}, \mathscr{H})$ симметрический оператор

$$
A=-i \frac{d}{d x}, \quad \operatorname{dom}(A)=W_{0}^{1,2}\left(\mathbb{R}_{-}\right) \oplus W_{0}^{1,2}\left(\mathbb{R}_{+}\right)=\left\{f \in W^{1,2}(\mathbb{R}): f(0)=0\right\} .
$$

Определим граничные тройки $\Pi_{j}=\left\{\mathscr{H}, \Gamma_{0}^{(j)}, \Gamma_{1}^{(j)}\right\}, j \in\{1,2\}$, для $A^{*}$, полагая

$$
\sqrt{2} \Gamma_{0}^{(1)} f:=[f(+0)-f(-0)], \quad \sqrt{2} \Gamma_{0}^{(1)} f:=i[f(+0)+f(-0)],
$$

и $\Gamma_{0}^{(2)}:=3^{-1 / 2} \Gamma_{0}^{(1)}, \Gamma_{1}^{(2)}:=\sqrt{3} \Gamma_{1}^{(1)}$. Соответствующие функции Вейля $M_{j}(\cdot), j \in\{1,2\}$, имеют вид

$$
M_{1}(z)=\left\{\begin{array}{ll}
i I_{\mathscr{H}}, & z \in \mathbb{C}_{+}, \\
-i I_{\mathscr{H}}, & z \in \mathbb{C}_{-},
\end{array} \quad M_{2}(z)= \begin{cases}3 i I_{\mathscr{H}}, & z \in \mathbb{C}_{+}, \\
-3 i I_{\mathscr{H}}, & z \in \mathbb{C}_{-} .\end{cases}\right.
$$

Полагая в $(10) B_{1}=-i I_{\mathscr{H}}$ и $B_{2}=i I_{\mathscr{H}}$, получаем аккумулятивное и диссипативное расширения $A_{B_{j}}, j \in\{1,2\}$, оператора $A$, которые даже не подобны. Действительно, $\sigma_{p}\left(A_{B_{1}}\right)=$ $\mathbb{C}_{-}, \sigma\left(A_{B_{1}}\right)=\overline{\mathbb{C}}_{-}$и $\sigma\left(A_{B_{2}}\right)=\mathbb{R}$.

В то же время

$$
\left(B_{1}-M_{1}(z)\right)^{-1}=-\frac{i}{2} \cdot I_{\mathscr{C}}=\left(B_{2}-M_{2}(z)\right)^{-1}, \quad z \in \mathbb{C}_{+} .
$$


Отметим еще, что оператор $A_{B_{2}}$ подобен самосопряженному оператору $A_{0}=-i d / d x$, $\operatorname{dom}\left(A_{0}\right)=W^{1,2}(\mathbb{R})$. В этом примере $A_{0}^{(1)}=A_{0}^{(2)}=: A_{0}$ и спектральная мера $E_{A_{0}}$ оператора $A_{0}$ спектрально эквивалентна мере $I_{\mathscr{H}} d t$.

\section{СПИСОК ЦИТИРОВАННОЙ ЛИТЕРАТУРЫ}

[1] В. И. Горбачук, М.Л. Горбачук, Граничные задачи для дифференциалъно-операторных уравнений, Наукова Думка, Киев, 1984. [2] V. A. Derkach, M. M. Malamud, J. Funct. Anal., 95:1 (1991), 1-95. [3] V.A. Derkach, M. M. Malamud, J. Math. Sci., 73:2 (1995), 141-242. [4] M. M. Malamud, V. I. Mogilevskii, Methods Funct. Anal. Topology, 8:4 (2002), 72-100. [5] M. Brown, J. Hinchcliffe, M. Marletta, S. Naboko, I. Wood, Integral Equations Operator Theory, 63:3 (2009), 297-320. [6] Yu. M. Arlinskii, S. Hassi, H. de Snoo, Operator Theory and Indefinite Inner Product Spaces, Oper. Theory Adv. Appl., 163, Birkhäuser Verlag, Basel, 2006, 23-54. [7] Д. З. Аров, М.А. Нудельман, Матем. сб., 193:6 (2002), 3-24. [8] В. Е. Лянце, О. Г. Сторож, Методы теории неограниченных операторов, Наукова Думка, Киев, 1983. [9] М. С. Бродский, Треугольные и жсордановы представления линейных операторов, Наука, М., 1969.

М. М. Маламуд

Поступило

Институт прикладной математики и

31.12 .2010

механики НАН Украины, г. Донецк

E-mail: mmm@telenet.dn.ua

\section{В. И. Могилевский}

Луганский национальный университет им. Тараса Шевченко

E-mail: vim@mail.dsip.net

С. Хасси

University of Vaasa, Финляндия

E-mail: sha@uwasa.fi 